\title{
RESEARCH
}

Open Access

\section{Therapeutic potential of small extracellular vesicles derived from lipoma tissue in adipose tissue regeneration - an in vitro and in vivo study}

Pengyu Hong ${ }^{1}$, Xiaoyang $\mathrm{Xu}^{1}$, Xin $\mathrm{Hu}^{2}$, Hao Yang ${ }^{1}$, Yue $\mathrm{Wu}^{1}$, Juan Chen ${ }^{1}$, Kun $\mathrm{Li}^{1 *}$ and Zhangui Tang ${ }^{1 *}$

\begin{abstract}
Objective: To explore the adipogenic effects of the small extracellular vesicles derived from the lipoma tissues (sEV$L T$ ), and to find a new cell-free therapeutic approach for adipose tissue regeneration.

Methods: Adipose tissue-derived stem cells (ADSCs) and small extracellular vesicles derived from the adipose tissues (sEV-AT) were isolated from human adipose tissue, while sEV-LT were isolated from human lipomatous tissue. ADSCs were characterized by using flow cytometric analysis and adipogenic and osteogenic differentiation assays. sEV was identified by electron microscopy, nanoparticle tracking, and western blotting. ADSCs were treated with sEV-LT and sEV-AT, respectively. Fluorescence confocal microscopy was used to investigate whether sEV-LT and sEV-AT could be taken by ADSCs. The proliferation and migration abilities and adipogenic differentiation assay of ADSCs were evaluated by CCK-8 assays, scratch test, and oil red O staining test, and the expression levels of adipogenic-related genes C/EBP- $\delta$, PPARY2, and Adiponectin in ADSCs were assessed by real-time quantitative PCR (RT-PCR). The sEV-LT and sEV-AT transplantation tubes were implanted subcutaneously in SD rats, and the neotissues were qualitatively and histologically evaluated at 2, 4, 8, and 12 weeks after transplantation. Hematoxylin and eosin (H\&E) staining was subsequently used to observe and compare the adipogenesis and angiogenesis in neotissues, while immunohistochemistry was used to examine the expression and the distribution of C/EBP-a, PPARy, Adiponectin, and CD31 at the 4th week.

\footnotetext{
*Correspondence: 406889138@qq.com; tangzhangui@aliyun.com

'Department of Oral \& Maxillofacial Surgery, Xiangya Stomatological Hospital \& School of Stomatology, Central South University, Changsha 410008, Hunan, China

Full list of author information is available at the end of the article
}

C C The Author(s). 2021 Open Access This article is licensed under a Creative Commons Attribution 4.0 International License, which permits use, sharing, adaptation, distribution and reproduction in any medium or format, as long as you give appropriate credit to the original author(s) and the source, provide a link to the Creative Commons licence, and indicate if changes were made. The images or other third party material in this article are included in the article's Creative Commons licence, unless indicated otherwise in a credit line to the material. If material is not included in the article's Creative Commons licence and your intended use is not permitted by statutory regulation or exceeds the permitted use, you will need to obtain permission directly from the copyright holder. To view a copy of this licence, visit http://creativecommons.org/licenses/by/4.0/. The Creative Commons Public Domain Dedication waiver (http://creativecommons.org/publicdomain/zero/1.0/) applies to the data made available in this article, unless otherwise stated in a credit line to the data. 
(Continued from previous page)

Results: The in vitro experiments showed that both sEV-LT and sEV-AT could be taken up by ADSCS via endocytosis. The scratch experiment and CCK-8 experiment showed that the migration area and proliferation number of ADSCs in sEV-LT group and sEV-AT group were significantly higher than those in the non-sEV group $(p<0.05)$. Compared with sEV-AT group, sEV-LT group had larger migration area and proliferation number of ADSCs $(p<0.05)$. Oil red $O$ staining and RT-PCR experiments showed that, compared with the non-sEVs group, the lipid droplets and the mRNA expression levels of adipogenesis-related genes PPARY2 and Adiponectin of ADSCs in sEVLT group and sEV-AT group were significantly upregulated $(p<0.05)$; however, there was no statistical significance in the expression level of C/EBP- $\delta(p>0.05)$. In addition, no significant difference in the amount of lipid droplets and adipogenesis-related genes between the sEV-LT groups and SEV-AT was seen ( $p>0.05)$. At 2, 4, 8, and 12 weeks, the adipocyte area and the number of capillaries in neotissues in the sEV-LT groups and sEV-AT groups were significantly increased compared with the Matrigel group $(p<0.05)$; however, there was no dramatic difference between sEV-LT groups and sEV-AT groups $(p>0.05)$. At the 4th week, neotissues in the sEV-LT groups and sEV-AT groups all showed upregulated expression of C/EBP-a, PPARY, Adiponectin, and CD31 protein, while neotissues in the Matrigel group only showed positive expression of CD31 protein.

Conclusions: This study demonstrated that SEV-LT exerted promotion effects on adipose tissue regeneration by accelerating the proliferation, migration, and adipogenic differentiation of ADSCs in vitro and recruiting adipocytes and promoting angiogenesis in vivo. The sEV-LT could serve as an alternative cell-free therapeutic strategy for generating adipose tissue, thus providing a promising application prospect in tissue engineering.

Keywords: Adipose tissue, Lipoma tissue, Small extracellular vesicles, Adipose tissue regeneration

\section{Introduction}

Soft tissue defects resulting from resection of tumors, as well as from trauma and congenital abnormalities, not only lead to disfigurement, but also impair functions, making adipose tissue restoration an urgent clinical need [1]. In 1893, Neuber first reported the use of autologous fat transplantation to successfully repair tissue defects; however, controversial results were reported in the limited therapeutic effect of this strategy, due to fat reabsorption, necrotic, liquefaction, and possible scar contracture in the donor sites [2, 3]. In more recent years, studies have shown that transplantation of adipose tissue-derived stem cells (ADSCs) can enhance adipose tissue regeneration via the paracrine actions of various cytokines and growth factors [4-6]. For example, our previous studies have demonstrated that fat grafts consisting of platelet-rich plasma and ADSCs constitute an ideal transplant strategy, which may result in decreased absorption and accelerated fat regeneration [7]. Although cell-based therapy has demonstrated the beneficial effects on adipose tissue regeneration, there are still lots of problems in the application of those mesenchymal stem cells (MSCs), such as immunogenicity, low viability, and potential tumorigenic feature $[8,9]$. Interestingly, recent works have demonstrated that paracrine factors significantly contribute to the therapeutic effect of stem cells on tissue repair [10]. In particular, extracellular vesicles (EVs) may play an important role in paracrine mechanisms and have attracted attention in basic research and clinical applications [11].

EVs are nano-sized membrane vesicles involved in intercellular communication, which have gained the most attraction as a potential and promising cell-free molecule used in clinical therapeutic applications [1113]. According to the guideline of MISEV2018 (Minimal information for studies of extracellular vesicles 2018), extracellular vesicles with size less than $200 \mathrm{~nm}$ were termed as sEVs (small extracellular vesicles) [11]. The growing evidence has proved that SEVs are secreted by a variety of cells and mediate local and systemic intercellular communication by transfer their contents into the target cells [14]. In general, sEVs contain messages from the original cell sources including bioactive proteins such as cytokines and growth factors, as well as lipids and nucleic acids (RNA and DNA), which modulate biological behaviors of the target cells $[15,16]$. Adipose tissue is an active endocrine organ that can secrete various factors to regulate adipogenesis via paracrine signals [17]. Previous studies have proved that sEVs derived from the adipose tissues (sEV-AT) are able to take part in a wide range of biological processes, especially for inducing adipogenic differentiation of ADSCs in vitro and promoting adipose tissue regeneration in vivo $[17,18]$.

Lipomas are common benign tumors of adipose tissues that originate in mesenchymal progenitors. Lipomas are usually treated when small in size; however, they sometimes can grow larger than $10 \mathrm{~cm}$ and can weigh over 1 $\mathrm{kg}$ [19]. Generally, most of the lipoma tissues after surgical resection are considered useless and used to be discarded. In clinic, intact lipoma tissues seem to be easier to obtain than normal adipose tissues, which are often fragmentized after liposuction. Studies have shown that a large number of adipocytes derived from lipoma 
tissues were strongly surrounded by Ki67+/CD34+ cells, indicating several altered biological activities such as proliferation, apoptosis, and stemness of those adipocytes $[20,21]$. Although several researches have indicated that lipoma tissue may have a faster rate of adipogenesis than normal adipose tissue, there is no study about the effect of sEVs derived from the lipoma tissue (sEV-LT) on adipose tissue regeneration. Therefore, in this study, we examined and analyzed sEV-LT. We observed the effects of sEV-LT on the proliferation, migration, and adipogenic differentiation of ADSCs. Furthermore, we explored the inductive effect of sEV-LT on adipose tissue regeneration in subcutaneous chamber models of SD rats. Collectively, our study provides a novel theoretical basis and a potential cell-free therapeutic strategy for adipose tissue regeneration.

\section{Methods}

\section{Animals and patients}

Before surgery, all patients were informed of the purpose and procedures of this study and agreed to offer their excised tissues. Written consent was obtained from all participants involved in this study. Human adipose tissues and subcutaneous lipoma tissues were intactly obtained from male patients (mean age $36.7 \pm 8.3$ years, age range $25-50$ years, $n=10$ ) by surgical excisions, who underwent skin transplantations at Xiangya Stomatological Hospital (Changsha, China). SPF Sprague-Dawley (SD) rats were purchased from the Department of Laboratory Animals of Central South University (Changsha, China).

\section{Isolation and culture of ADSCs}

ADSCs were obtained from human subcutaneous adipose tissues. The fresh adipose tissues were washed three times with sterile phosphate-buffered saline (PBS) containing $1 \%$ penicillin and streptomycin, chopped by sterile operation scissors into small pieces $\left(1-2 \mathrm{~mm}^{3}\right)$, and digested with $3 \mathrm{mg} / \mathrm{ml}$ type I collagenase (Sigma, Germany) for $40 \mathrm{~min}$ at $37^{\circ} \mathrm{C}$ with shaking and then centrifuged at $1400 \mathrm{rpm}$ for $7 \mathrm{~min}$. The sediments were resuspended and expanded in the culture medium consisting of DMEM/F12 medium (BI, Israel), 10\% fetal bovine serum (FBS, BI, Israel), 1\% penicillin/streptomycin, and $2 \mathrm{mM} \mathrm{L}$-glutamine at $37{ }^{\circ} \mathrm{C}$ with $5 \% \mathrm{CO}_{2}$.

\section{Characterization of ADSCs}

The ADSCs at passage 3 were harvested and counted. Approximately $1 \times 10^{5}$ cells were washed and labeled with fluorescence-conjugated antibodies (Biolegend, USA) (CD105-PE, CD90-PE, CD73-PE, CD34-PE, CD14$\mathrm{PE})$ at room temperature for $30 \mathrm{~min}$. Isotype control IgG1 and IgG2a were used to stain the cells as a control. After being washed with PBS twice, the fluorescence of
ADSCs were observed. For adipogenic or osteogenic differentiation, the cells were seeded in standard 6-well tissue culture plates $\left(1.5 \times 10^{5}\right.$ cells per well and incubated with adipogenic differentiation medium (Cyagen, China) for 1 week or with osteogenic differentiation medium (Cyagen, China) for 2 weeks, respectively. Then, the induced cells were stained separately with Oil Red O for $30 \mathrm{~min}$ to assess adipogenic differentiation or with Alizarin Red S (Cyagen, China) for $5 \mathrm{~min}$ at room temperature to visualize osteogenic differentiation.

\section{Isolation of small extracellular vesicles}

Patients' adipose tissues and lipoma tissues were minced into small pieces and transferred into a Celstir spinner flask (Wheaton) supplemented with Serum-free Dulbecco's modified eagle medium/f12 (DMEM/F12) and 1\% penicillin/streptomycin, respectively. The tissues were cultured at $37^{\circ} \mathrm{C}$ and a rotational speed of $100 \mathrm{rpm}$ for 2 days. The debris of tissues and cells were removed by centrifugation (2000g, $30 \mathrm{~min})$. An additional centrifugation in Amicon ${ }^{\circ}$ Ultra-50 Centrifugal Filter Units with Ultracel-3 membrane (3000Mw cutoff membrane, Millipore) at $5000 \mathrm{~g}$ for $30 \mathrm{~min}$ was applied to concentrate lipoma tissue extract (LTE) and adipose tissue extract (ATE). Then, the ATE and LTE were mixed with the Total Exosome Isolation ${ }^{\mathrm{mi}}$ reagent (Life Technologies) at $4{ }^{\circ} \mathrm{C}$ overnight and a final ultracentrifugation step was performed at 10,000 $\mathrm{g}$ for $1 \mathrm{~h}$ at $4{ }^{\circ} \mathrm{C}$. The obtained pellet was resuspended in $400 \mu \mathrm{l}$ of PBS and stored at $-80^{\circ} \mathrm{C}$ with known concentration determined by using the Pierce BCA protein assay kit (KeyGEN, China).

\section{Characterization of small extracellular vesicles}

The ultrastructure and size distribution of the sEV-LT and SEV-AT were analyzed by a transmission electron microscopy (TEM) (FEI Tecnai G2 Spirit, USA) and the ZetaView $^{\circ}$ system (Particle Metrix, Germany), respectively. Twenty-microgram vesicles was dissolved in RIPA Lysis Buffer (KeyGEN, China) and separated on polyacrylamide gels, blotted onto a nitrocellulose membrane. The specimens were then incubated with a primary antibody CD9 (1:1000, Abcam, ab92726), CD63 (1:1000, Abclonal, a5271), and TSG101 (1:1000, Proteintech, 28283-1-ap) at $4{ }^{\circ} \mathrm{C}$ overnight and followed by horseradish peroxidase-coupled secondary antibody for $1 \mathrm{~h}$ at room temperature. The labeled protein markers were visualized using ImageQuant LAS 4000 mini (GE Healthcare).

\section{Small extracellular vesicles labeling and cellular uptake}

The sEVs were labeled with a membrane-labeling dye $\mathrm{DiO}$ (Invitrogen, USA) and then washed and resuspended in serum free DMEM/F12. After that, ADSCs were co-cultured with DiO-labeled vesicles for $6 \mathrm{~h}$, 
washed with PBS three times, fixed in $4 \%$ paraformaldehyde, stained with phallotoxins (Invitrogen, USA), washed with PBS three times, counterstained with 4',6diamidino-2-phenylindole (DAPI, Sigma, Germany), and washed with $\mathrm{PBS}$ three times and imaged by a confocal microscopy (Olympus FV1000, Japan).

\section{Cell migration assay}

The effects of sEV-LT and sEV-AT on ADSCs migration were evaluated in a scratch assay. ADSCs were seeded and cultured in 6-well plates at a seeding density of $2 \times$ $10^{5}$ cells/well. When the cell confluence reached $90 \%$, the medium was replaced with DMEM/F12 after washing with PBS twice, the confluent cell monolayer was scratched using a sterile $1000 \mu \mathrm{l}$ pipette tip, and the cells were washed with PBS. The sEV-LT $(40 \mu \mathrm{g} / \mathrm{ml}, 1.40 \times$ $10^{9}$ particles $\left./ \mathrm{ml}\right)$, sEV-AT $\left(40 \mu \mathrm{g} / \mathrm{ml}, 2.55 \times 10^{9}\right.$ particles $/ \mathrm{ml}$ ), and an equal volume of PBS were added to the wells, respectively. Images were recorded at 0,12 , and $24 \mathrm{~h}$ after the monolayers were scratched. The migration area was measured by using ImageJ software and assessed as follows: migration area $(\%)=(\mathrm{A} 0-\mathrm{An}) /$ A0 $\times 100$ (A0 represents the initial wound area $(t=0 \mathrm{~h}$ ) and An represents the residual area of the wound at the time of measurement $(t=\mathrm{nh})$ ).

\section{Cell proliferation assay}

The growth of ADSCs was determined by a Cell Counting Kit-8 (CCK-8, KeyGEN, China) assay. The ADSCs were cultured in 96-well plates with a seeding density of 1500 cells/well and the medium was replaced with PBS. The cells were cocultured with sEV-LT $(40 \mu \mathrm{g} / \mathrm{ml}$, $1.40 \times 10^{9}$ particles $\left./ \mathrm{ml}\right)$, sEV-AT $\left(40 \mu \mathrm{g} / \mathrm{ml}, 2.55 \times 10^{9}\right.$ particles $/ \mathrm{ml}$ ), or an equal volume of PBS. At $0,1,2,3,4$, and 5 days, $10 \mathrm{~mL}$ cell counting solution was added into each well and incubated at $37^{\circ} \mathrm{C}$ for $1 \mathrm{~h}$. The optical density (OD) was measured at $450 \mathrm{~nm}$ using a microplate reader (BioTek, USA).

\section{Adipogenic differentiation of ADSCs}

The ADSCs at passage 3 were seeded at $2 \times 10^{5}$ cells per well into six-well plates, cultured for $24 \mathrm{~h}$, then rinsed with PBS and incubated with $2 \mathrm{ml}$ of one of four different culture media for up to 14 days. The media used were as follows: (1) basal medium (DMEM/F12 supplemented with $10 \%$ FBS) as a negative control, (2) basal medium supplemented with sEV-LT $(40 \mu \mathrm{g} / \mathrm{ml}, 1.76 \times$ $10^{9}$ particles $/ \mathrm{ml}$ ), (3) basal medium supplemented with sEV-AT $\left(40 \mu \mathrm{g} / \mathrm{ml}, 2.69 \times 10^{9}\right.$ particles $\left./ \mathrm{ml}\right)$, and (4) adipogenic medium (DMEM/F12 supplemented with $10 \%$ FBS, $2 \mathrm{mM}$ insulin $+0.5 \mathrm{mM}$ isobutylmethylxanthine + $0.1 \mu \mathrm{M}$ dexamethasone $+5 \mu \mathrm{M}$ rosiglitazone) as a positive control. The medium was changed every 3 days. The expression of adipogenic genes were analyzed by real- time quantitative PCR (RT-PCR) after 7 days of induction. After 14 days culturing, adipogenic differentiation was determined by the Oil Red O (Cyagen, China) staining. The Oil Red O in cells was extracted with $100 \%$ isopropanol for $15 \mathrm{~min}$. The absorbance was measured at $520 \mathrm{~nm}$ with a microplate reader (BioTek, USA).

\section{Real-time PCR analysis}

The TRIzol ${ }^{\circ}$ reagent (Invitrogen, USA) was used to extract total RNA, which was reverse transcribed into cDNAs using the Revert Aid First Strand cDNA Synthesis Kit (Thermo Scientific, USA). The synthesized cDNAs were amplified with SYBR Premix ExTaq (TaKaRa Biotechnology, Japan) using a RT-PCR System (Biometra Tone, Germany). The PCR cycling parameters were $95^{\circ} \mathrm{C}$ for $2 \mathrm{~min}, 44$ cycles of $95^{\circ} \mathrm{C}$ for $5 \mathrm{~s}$, and $60^{\circ} \mathrm{C}$ for $30 \mathrm{~s}$ (the primers used for RT-PCR are shown in Table 1).

\section{Adipose tissue regeneration experiments in an animal model}

All operations were performed on 8-week-old SD rats $(200 \pm 20 \mathrm{~g}, n=16)$ under general anesthesia (1\% pentobarbital sodium, $10 \mathrm{~mL} / \mathrm{kg}$, intraperitoneal injection). A silicone tube with an internal diameter of $5.0 \mathrm{~mm}$ and a height of $5.0 \mathrm{~mm}$ was subcutaneously implanted into the longitudinal incision (about $4 \mathrm{~cm}$ ) on the back of rats. The incision was closed with 4/0 nylon suture. Each rat was implanted with 3 tubes: (1) $100 \mu \mathrm{L}$ Matrigel (Corning, USA) alone, (2) $100 \mu \mathrm{L}$ Matrigel containing $100 \mu \mathrm{g}$ sEV-LT by injection, and (3) $100 \mu \mathrm{L}$ Matrigel containing $100 \mu \mathrm{g}$ sEV-AT by injection. Rats were sacrificed at 2,4 , 8 , and 12 weeks, respectively ( $n=4$ per time point), and their implanted tubes and contents were harvested and analyzed for further investigation.

\section{Histology}

The excised neo-tissue samples from the silicone tubes were fixed in $10 \%$ formalin, dehydrated with a graded alcohol series, and embedded in paraffin. Sections with a thickness of $4 \mu \mathrm{m}$ were stained with hematoxylin and

Table 1 Primers used for RT-PCR

\begin{tabular}{ll}
\hline Gene & Primers \\
\hline PPARY2 & 5'-GCCCTTTGGTACTTTATGGAG-3' \\
& GCAGCAGGTTGTTTGGATG \\
C/EBP- $\delta$ & CTGCCATGTATGACGACGAGAG \\
& CGCTITGTGATTGTGTTGAAG \\
ADIPONECTIN & CGTTCTCTTCACCTACGACCAGT \\
& ATTGTTGTCCCCTTCCCCATAC \\
GAPDH & TCAACGGCACAGTCAAGG \\
& ACCAGTGGATGCAGGGAT \\
\hline
\end{tabular}


eosin (H\&E). Images of the histologic sections were examined microscopically (Leica, Germany) at a magnification of $\times 100$. To quantitatively analyze the area of intact adipocytes and the number of mature capillaries, images in 8 random fields per section from each group $(\times 100$ magnification) were examined by using ImageJ software. Immunochemical staining for C/EBP- $\alpha$ (Bioss, China, cat.bs1630R), PPARY (Bioss, China, cat.bs4888R), Adiponectin (Bioss, China, cat.bs0471R), and CD31 (Sino Biological, China, cat.50408-T16) was performed to determine the extent of adipose tissue and blood vessel formation in new growth tissues at the 4th week.

\section{Statistical analysis}

Each experiment was repeated at least three times. Data were expressed as mean \pm standard (SD). Statistical analysis was performed with a paired Student's $t$-test. A probability $(p)$ value $<0.05$ was considered statistically significant.

\section{Results}

\section{Characterization of ADSCs, sEV-LT, and sEV-AT}

The morphology of ADSCs at passage 3 exhibited a spindle-like shape which is typical for mesenchymal stem cells under the inverted microscope (Fig. 1a). ADSCs also displayed adipogenic differentiation and osteogenic differentiation abilities, which were demonstrated by Oil red O staining for adipocytes (Fig. 1b) and Alizarin Red S staining for osteoblasts (Fig. 1c), respectively. Flow cytometry analysis (Fig. 1d) showed that these cells were highly positive for CD105, CD90, and CD73, while negative for CD34 and CD31. These results were consistent with previous studies in the characterizations of ADSCs [7]. Therefore, all these data unequivocally confirmed that ADSCs were successfully isolated from human normal adipose tissues.

We then isolated sEV-LT and sEV-AT from the tissue extracts. The cup-shaped morphology of the extracellular vesicles was observed by TEM (Fig. 1e). The size of sEV-LT and sEV-AT were directly tracked by using a Particle Metrix Analyzer named the ZetaView system. The average size of sEV-LT and sEV-AT were $121.3 \mathrm{~nm}$ and $121.1 \mathrm{~nm}$, respectively (Fig. 1f). Western blots also showed that the exosomal markers CD9, CD63, and TSG101 (Fig. 1g) were all expressed in SEV-LT and sEVAT. These results indicated that sEV-LT and sEV-AT were successfully isolated as they were consistent with the defining characteristics of sEV [14].

The biological responses of ADSCs to sEV-LT and sEV-AT To explore whether sEV-LT and sEV-AT could be internalized by ADSCs, ADSCs were co-cultured with the DiO-labeled extracellular vesicles for $6 \mathrm{~h}$ and then observed by the fluorescence confocal microscopy. The
DiO-labeled (green) sEV-LT and sEV-AT were seen to localize predominantly at the perinuclei region after entering cells, indicating that endocytosis might be the main mechanism through which ADSCs internalized extracellular vesicles (Fig. 2a).

In order to preferably examine the effects of sEV-LT on the migration and proliferation of ADSCs, we used sEV-AT for comparison. It was clear that sEV-LT and sEV-AT promoted the migration of ADSCs at different time points. The results from the scratch closure test demonstrated that the migration of ADSCs increased after $12 \mathrm{~h}$ and $24 \mathrm{~h}$ in the presence of sEV-LT and sEVAT compared to the migration in the control group. At $12 \mathrm{~h}$, cell migration of sEV-AT group was higher than that of sEV-LT group. However, cell migration of sEVLT group was higher than that of sEV-AT group at $24 \mathrm{~h}$ (Fig. 2b-c). Likewise, CCK-8 analysis showed that compared to the control group, sEV-LT and sEV-AT at a concentration of $40 \mu \mathrm{g} / \mathrm{mL}$ both increased the proliferation of ADSCs, while sEV-LT had a much stronger promotion effect than sEV-AT (Fig. 2d).

To examine the effects of sEV-LT on adipogenesis, ADSCs were continuously co-cultured with sEV-LT $(40 \mu \mathrm{g} / \mathrm{ml})$ for 14 days. Lipid droplets were showed when ADSCs were treated with both sEV-LT and sEV-AT after 14 days induction (Fig. 2e). Adipogenesis was further determined by Oil Red $\mathrm{O}$ staining. The results showed that the OD value of extracted Oil Red O was much higher in both sEV-LT group and sEV-AT group than that in the negative control group. However, there was no significant difference in OD value between sEVLT group and sEV-AT group (Fig. 2f). Similarly, RTPCR revealed that the mRNA levels in SEV-LT group and sEV-AT group for the adipogenic genes encoding PPAR 2 and ADIPONECTIN were remarkably elevated on day 7 compared to that in the negative control group, while there was no significant difference in mRNA expression between sEV-LT group and sEV-AT group (Fig. 2g). Moreover, the expression of mRNA encoding C/EBPS remained at a similarly low level (Fig. 2g).

\section{sEV-LT promoted adipose tissue regeneration in vivo Gross observations and weights}

In order to explore the effects of sEV-LT on adipose tissue regeneration in vivo, Matrigel mixed with sEV-LT and sEV-AT was injected into the silicone tube and kept at $37^{\circ} \mathrm{C}$ for $30 \mathrm{~min}$ to become solid, then transplanted into the back of SD rats, respectively, while the Matrigel alone served as the control (Fig. 3a). The gross appearance of tubes was shown and their appearance at 2, 4, 8, and 12 weeks revealed active angiogenesis and adipogenesis in the sEV-LT group and sEV-AT groups, since there were a few growths of capillaries and adipose-like tissue from host into the tubes, while in control group, 

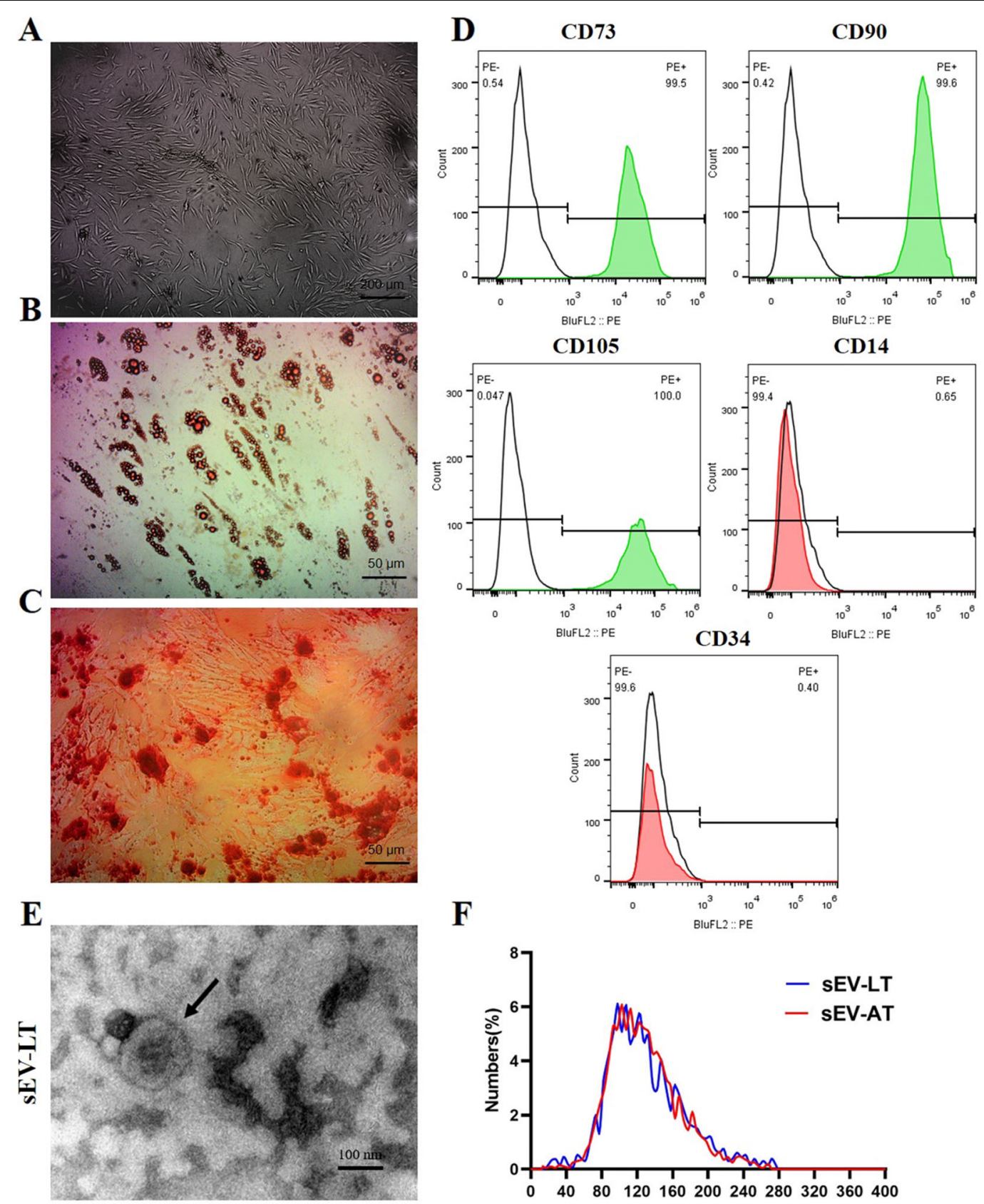

F
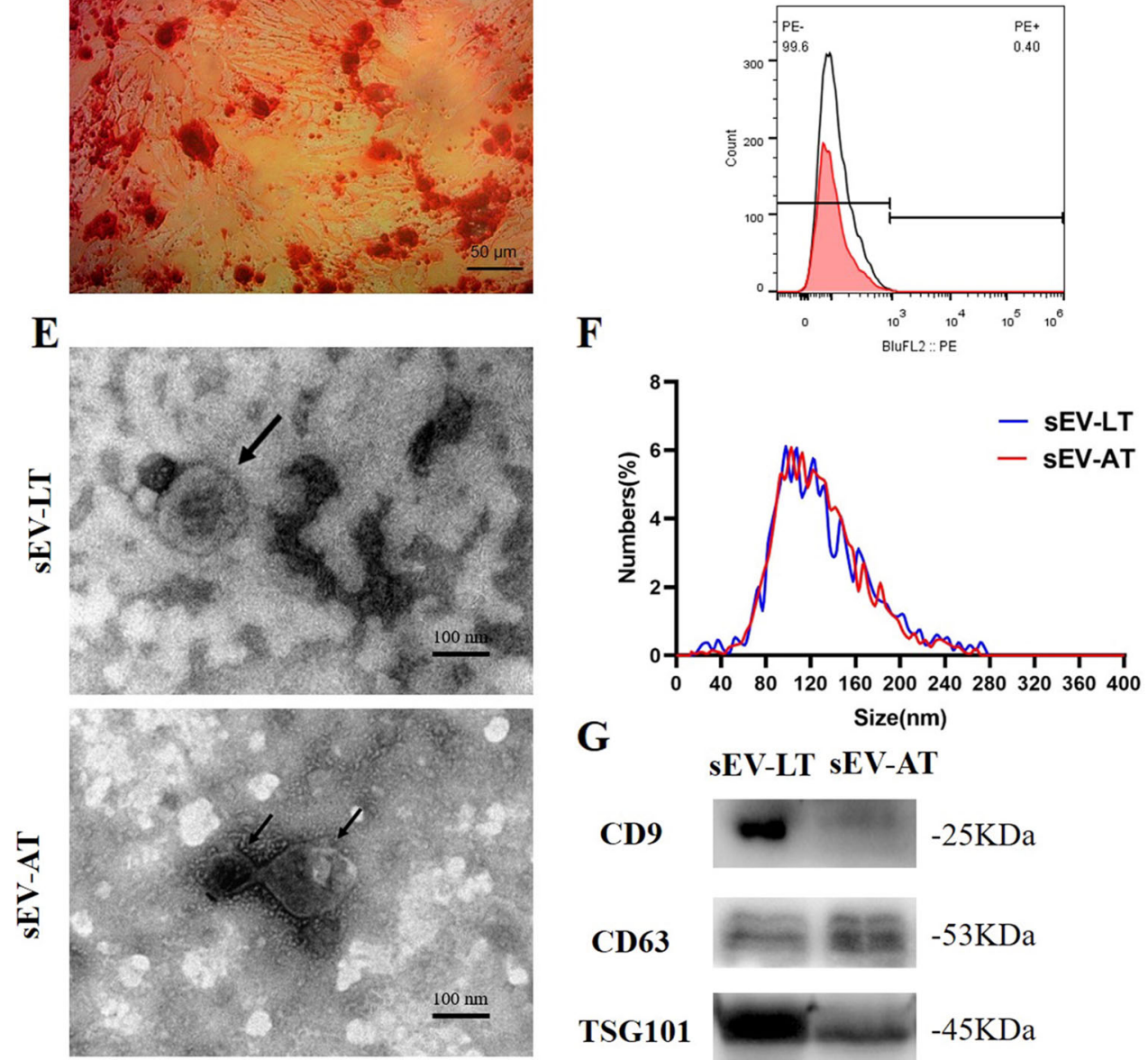

Fig. 1 (See legend on next page.) 
(See figure on previous page.)

Fig. 1 Characterization of ADSCS, SEV-LT and SEV-AT. a ADSCS exhibited the typical spindle-shaped morphology, as shown in the picture. Scale bar $=200 \mu \mathrm{m}$. b Representative photographs of Oil red O staining for adipocytes. Scale bar $=50 \mu \mathrm{m}$. c Representative photographs of Alizarin Red S staining for osteoblasts. Scale bar $=50 \mu \mathrm{m}$. d Flow cytometry analysis showed that these cells were highly positive for CD105, CD90, and CD73 but negative for CD34 and CD31. Abbreviations: PE, phycoerythrin. e The ultrastructure of sEV-LT and sEV-AT under transmission electron microscopy. Scale bar $=100 \mathrm{~nm}$. $\mathbf{f}$ The size distribution profile of sEV-LT and sEV-AT by dynamic light scattering. $\mathbf{g}$ The expression of the sEV markers CD9, CD63, and TSG101 was confirmed by immunoblotting

tubes filled with Matrigel were mainly encapsulated by integral fibrous fascia (Fig. 3b, c). The neotissues in the tubes were harvested and the macroscopic images at all time points were taken (Fig. 3d). The weights of neotissues were systematically calculated at each time-point and found gradually increased in all groups. Furthermore, the weights of neotissues were significantly increased in the sEV-LT group and sEV-AT group compared to the control groups, but there was no significant difference in that between sEV-LT group and sEV-AT group (Fig. 3e).

\section{Histological observations and newly formed capillaries}

Neotissues from all groups were stained with hematoxylin and eosin $(\mathrm{H} \& \mathrm{E})$, and the quantitative comparisons of the newly formed areas of adipocytes and capillary numbers have been carried out. In the sEV-LT group and sEV-AT group, light microscopy revealed that immature adipocytes with irregular morphologies and small diameters showed up after 2 weeks' transplantation, and neovascularization was seen beside the adipocytes, while no obvious adipocytes grew in the Matrigel group (Fig. 4a). At the 4th week, the light microscopy showed that most of the Matrigel contained in each group had been absorbed completely, and an increased large number of regular adipocytes and capillaries were seen in the SEV-LT group and SEV-AT group (Fig. 4ac). At 8 and 12 weeks, the adipocyte areas and the numbers of capillaries observed in the sEV-LT group and sEV-AT group decreased, while the granulation and fibrous tissue areas gradually increased (Fig. $4 \mathrm{a}-\mathrm{C}$ ). Although some of the adipocytes were replaced by fibroblasts and granulosa cells, the remaining were maintained for 12 weeks and there still had significant increase in adipose tissue in the sEV-LT group and sEVAT group than the Matrigel groups (Fig. 4b, c).

\section{Immunohistochemistry}

Furthermore, we used immunohistochemistry to ascertain the adipogenesis and angiogenesis effects of sEVLT. Neotissue sections at the 4th week were stained with antibodies against C/EBP- $\alpha$, PPAR $\gamma$, Adiponectin, and CD31, which were all positively expressed in the cells of sEV-LT group and sEV-AT group. However, in the Matrigel group, only positive expression of CD31 protein was found. In addition, we found that many adipocytes and endothelial cells were positively stained near the blood vessels, while those cells located at a distance from the blood vessels were negative (Fig. 5).

\section{Discussion}

Previous studies in cell-free therapeutic approaches for adipose tissue regeneration have demonstrated certain effects to contribute to new adipose tissue development, including using synthetic or natural biomaterial scaffolds loaded with adipogenic growth factors, or providing a microenvironment suitable for originally existing cells in the body to migrate, proliferate, and differentiate to form adipose tissues [22, 23]. However, problems such as immune rejection responses or potential side effects of high growth factor concentrations may make it difficult to apply in clinic extensively. In recent studies, researchers have found and confirmed that cell-free adipose tissue extract (ATE) could effectively induce adipogenesis and angiogenesis [5, 24]. Furthermore, sEV-AT, as an indispensable component of ATE, has also been found that it could modulate proliferation, migration, and differentiation of the target cells by transferring functional proteins, mRNAs, and miRNAs $[16,25]$. For example, sEVAT under hypoxic conditions might promote lipid stimulation in 3T3-L1 adipocytes by increasing the levels of lipogenic enzymes, including fatty acid synthase (FASN), glucose-6-phosphate dehydrogenase (G6PD), and acetyl-CoA carboxylase (ACC), which might contribute to adipose tissue homeostasis or dysfunction [26]. In addition, Zhang et al. [17] found that sEV-AT could induce adipogenesis differentiation through a mechanism involving transfer of miR-450a-5p. Meanwhile, miR-450a-5p could promote adipogenesis through repressing expression of WISP2 by targeting its 3 ' untranslated region. To conclude, from a perspective of paracrine effect, sEV-AT could be delivered into target cells and could give rise to adipogenesis ultimately. Lipoma tissues, as adipose tissues of benign tumors, were always defined as useless tissues in the application of regenerative medicine and tissue engineering [27]. Previous studies basically focused on lipoma-derived stem cells; however, the exact biological functions of sEV-LT are still needed to be found and determined [28, 29].

Our study was among the first giving details in the characteristics and adipogenic analysis of sEV-LT and comparison with sEV-AT from human. In our studies, 


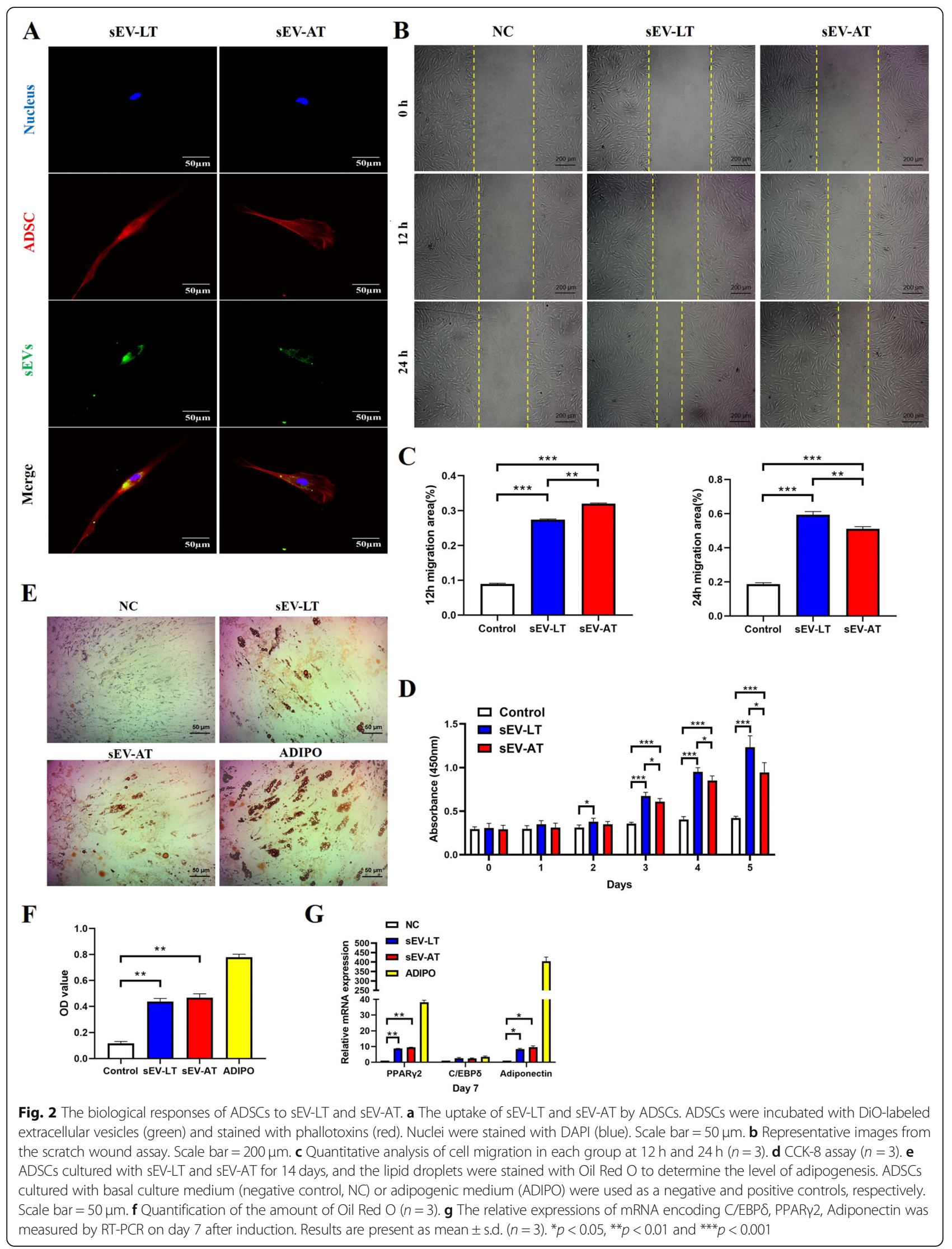




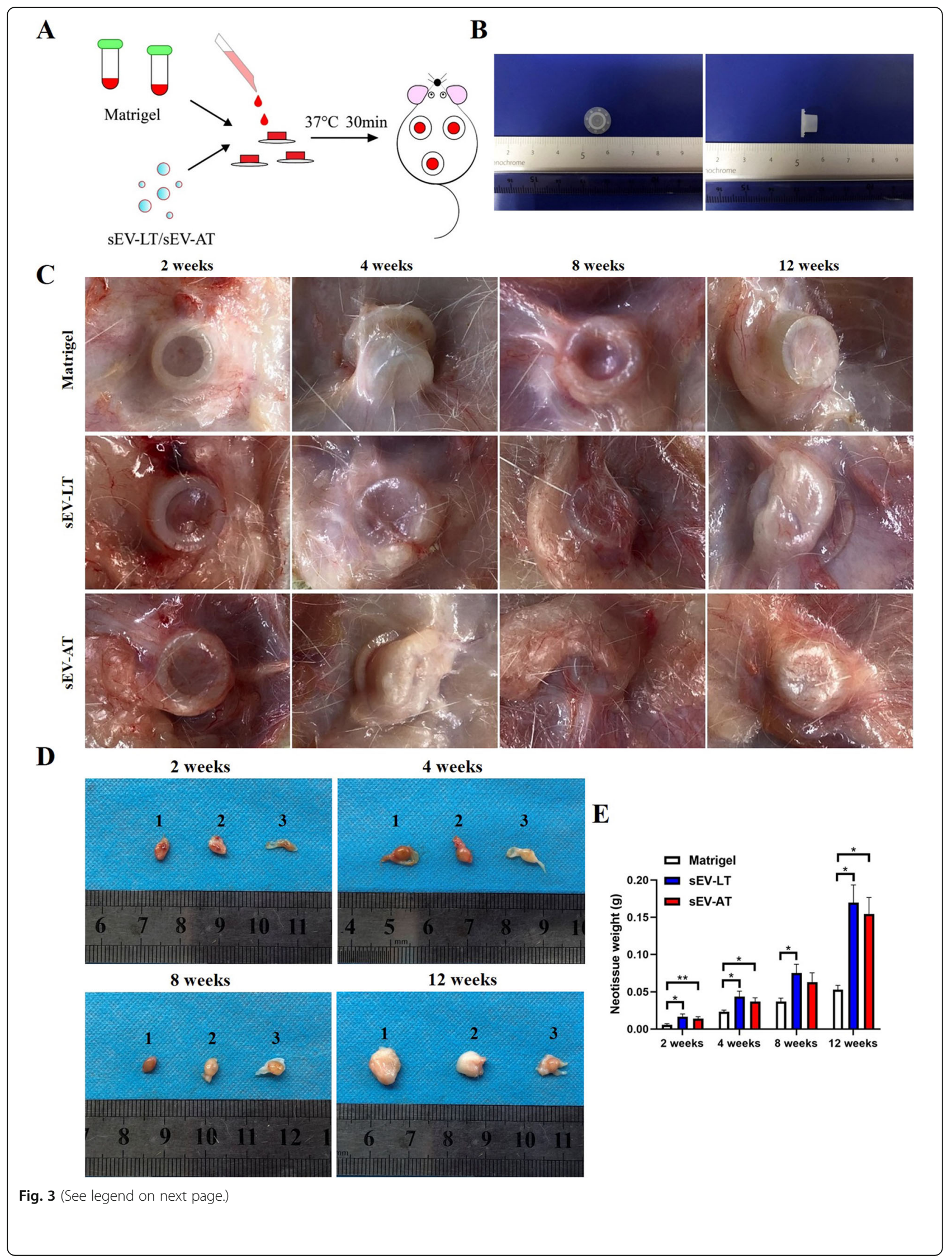


(See figure on previous page.)

Fig. 3 sEV-LT promoted neotissue formation in vivo. a The schematic view of the experimental operation process. b The implanted silicone tubes, with an internal diameter of $5.0 \mathrm{~mm}$ and a height of $5.0 \mathrm{~mm}$. c The gross observation of the implanted Matrigel mixed with SEV-LT and sEV-AT within the tube at weeks 2, 4, 8, and 12 after transplantation. $\mathbf{d}$ Dimensions of neotissues at weeks 2, 4, 8, and 12 after transplantation. (1) sEV-LT. (2) sEV-AT. (3) Matrigel group. e Weight comparisons of neotissues at weeks 2, 4, 8, and 12 after transplantation $(n=4)$.

${ }^{*} p<0.05,{ }^{* *} p<0.01$

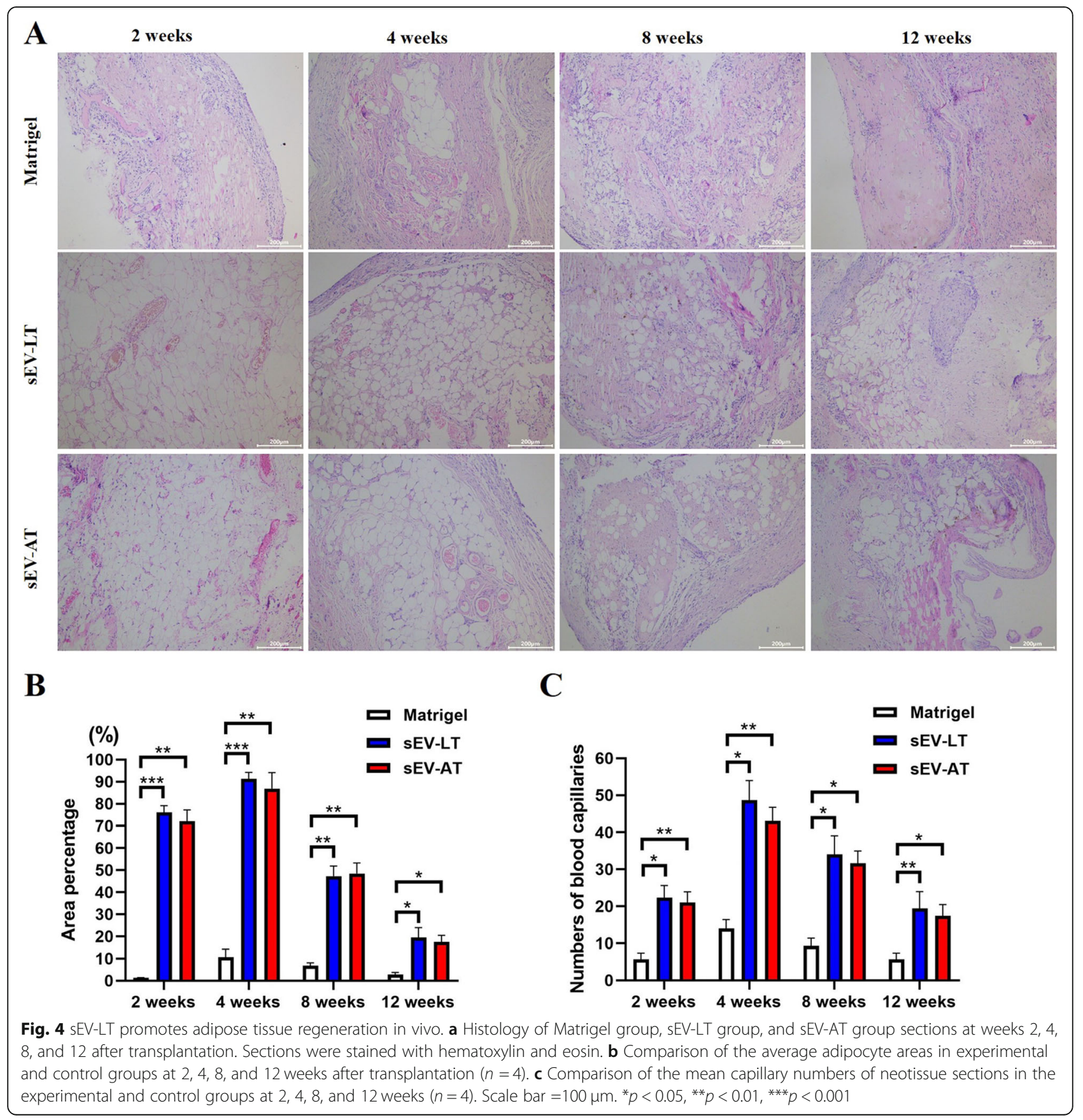



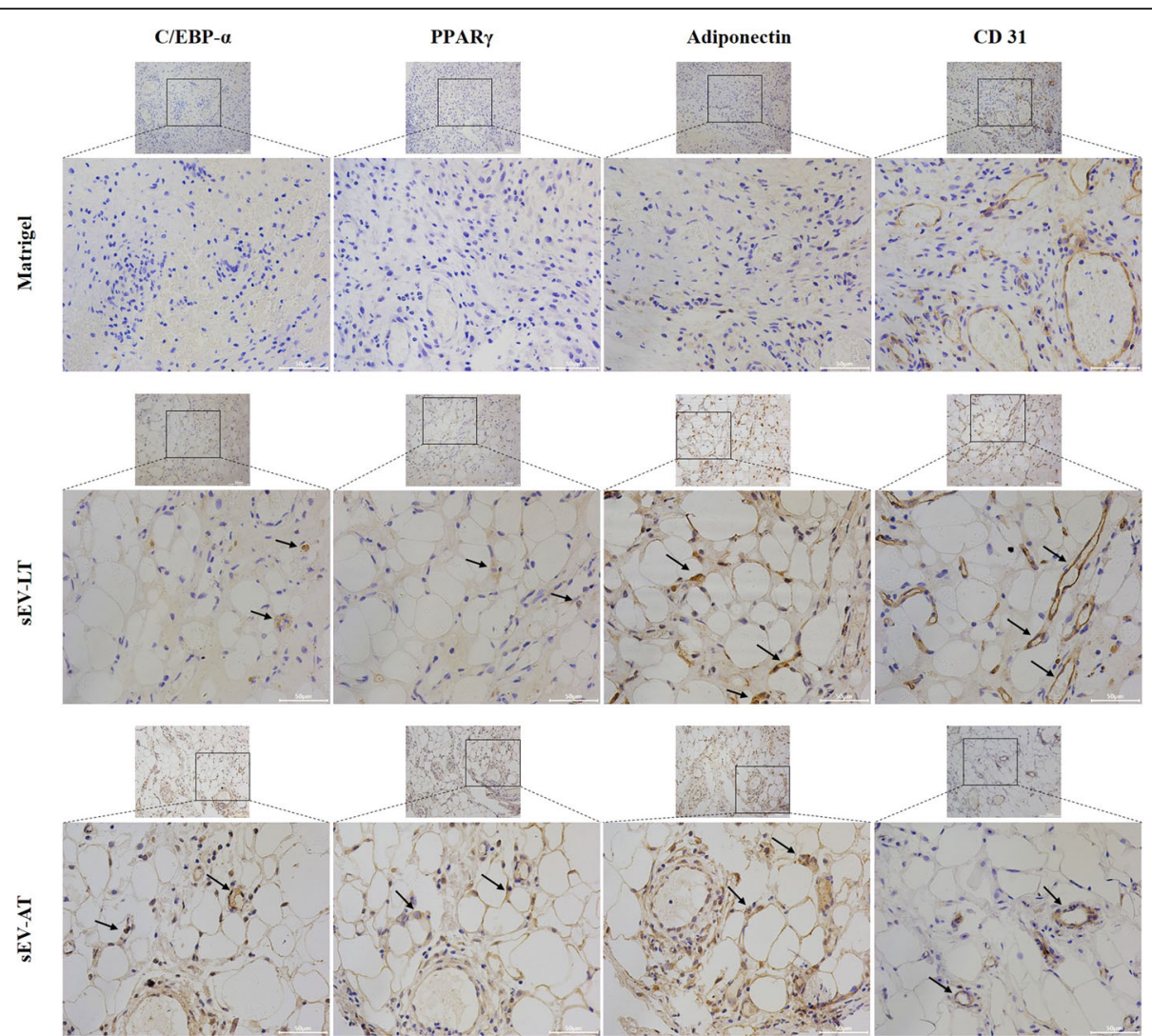

Fig. 5 Immunohistochemistry of neotissue sections in the experimental and control groups at week 4 after transplantation. Representative photographs of C/EBPa, PPARY, Adiponectin, and CD31 immunostaining in the Matrigel group, sEV-LT group, and sEV-AT group, respectively. The black arrows indicated the positive stained protein. Scale bar $=50 \mu \mathrm{m}$

we showed evidence that lipoma tissue could release sEV and ADSCs could uptake both sEV-LT and sEV-AT in a form of endocytosis. Furthermore, we found that sEV-LT and sEV-AT were able to effectively promote the proliferation, migration, and adipogenic differentiation of ADSCs. Compared with sEV-AT, sEV-LT had a stronger ability to promote the proliferation and migration of ADSCs, while there was no significant difference in inducing adipogenic differentiation of ADSCs. These results indicated that the local progressive growth of the lipoma tissue might be related to the increasing migration and proliferation of the surrounding ADSCs induced by SEV-LT. In fact, previous studies have also confirmed that EVs secreted by terminally differentiated cells can influence biological functions of stem cells [30-32]. For instance, EVs from osteoblasts, vascular endothelial cells, and skeletal muscle cells were found to be involved in the regulation of osteogenic differentiation, angiogenic differentiation, and myogenic differentiation of stem cells, respectively [30-32]. Once the stem cells differentiate into mature cells, they, in turn, regulate stem cells to differentiate through EVs; thus, a positive-feedback loop mechanism is formed during differentiation. Therefore, both sEV-LT and sEV-AT presented similar effects on ADSCs. However, we observed that sEV-LT showed stronger effects in promoting proliferation and migration of ADSCs than sEV-AT; these findings should be related to the differences in the functional proteins and miRNA and other genetic materials contained in the two kinds of $\mathrm{sEV}$, which may be associated with the core reason for the tumorigenicity of lipoma tissue.

In order to further investigate the potential functions of sEV-LT in adipose tissue regeneration, we transplanted SEV-LT into subcutaneous chamber models of $\mathrm{SD}$ rats and observed the effects over a long-term period of 12 weeks, which was sufficient for complete adipogenesis and neotissue stabilization. We found that capillaries were first visible at 2 weeks on the surface of the silicone tubes in the sEV-LT group and sEV-AT groups, while none of that were seen on the surface of the cannula in the Matrigel group. At 2, 4, 8, and 12 weeks, the adipocytes were detected under the microscope in the sEV-LT and SEV-AT groups and mainly appeared around the newly formed blood vessels. Compared with the Matrigel group, the adipocyte areas and the number of capillaries 
in the sEV-LT and sEV-AT groups were increased and statistically significant at all time points. Collectively, these results showed that the sEV-LT were able to promote adipogenesis and angiogenesis in vivo and the positive regulation relationship between adipogenic differentiation and angiogenesis, which was consistent with previous studies. It has been shown that the increased resorption of a fat graft is often related to debilitated and weakening neovascularization [33]. Vascular-derived factors regulate the metabolism and accumulation of adipose tissue by affecting the formation and reconstruction of adipose tissue vascular network [34]. Due to the close relationship between adipogenesis and angiogenesis, we used immunohistochemistry to analyze the expression of adipogenesis-related proteins PPAR $\gamma, \mathrm{C} /$ EBP- $\alpha$, and Adiponectin and angiogenesis-related protein CD31 in each group at 4 weeks. It was found that all these proteins were positively expressed in the sEVLT and sEV-AT groups, and adipogenesis-related proteins were mostly aggregated and expressed in adipocytes around capillaries. In contrast, they were relatively less expressed in adipocytes far away from microvessels. These results also confirmed the positive regulatory relationship between adipogenesis and angiogenesis.

However, along with the decrease of blood vessels, the adipocytes recruited in the sEV-LT and sEV-AT groups began to decrease and were replaced by fibroblasts and granular cells at 8 and 12 weeks. We hypothesized the main reason could be related to the rats' immune responses to those silicone tubes and limited quantities of EVs which gradually exhausted. Nevertheless, from a long-term perspective, the newly formed adipose tissue in sEV-LT could still display a certain vitality till 12 weeks, which indicated a therapeutic potential of sEVLT in fat regeneration and tissue engineering.

There are some limitations of our studies, and several problems must be solved before the results can be applied in the clinic setting. For instance, although we have verified the adipogenesis and angiogenesis effects of sEV-LT, the exact proteins or miRNAs involved in sEVLT-mediated differentiation are still needed to be determined in the future. Another limitation is that we need to further evaluate and confirm the tumorigenicity and biosafety of sEV-LT, since they are extracted from tumor tissue. Moreover, the number of SD rats in the study was too small; a larger sample size should be included in the future study. Additional studies will be performed in the future; however, our studies have provided a potential new method for adipose tissues regeneration and these findings will direct better clinical treatment.

\section{Conclusion}

In this study, to the best of our knowledge, we provided direct evidence that sEV-LT exerted promotion effects on adipose tissue regeneration by accelerating the proliferation and migration and adipogenic differentiation of ADSCs in vitro, recruiting adipocytes and promoting angiogenesis in vivo for the first time. Notably, the neotissue induced by sEV-LT could maintain viability lasting for 12 weeks. Therefore, sEV-LT could serve as an alternative cell-free therapeutic strategy for generating adipose tissue, providing a promising application prospect in tissue engineering.

\section{Abbreviations}

ADSCs: Adipose tissue-derived stem cells; ACC: Acetyl-CoA carboxylase; ATE: Adipose tissue extract; H\&E: Hematoxylin and eosin; FASN: Fatty acid synthase; G6PD: Glucose-6-phosphate dehydrogenase; LTE: Lipoma tissue extract; MSCs: Mesenchymal stem cells; PBS: Phosphate-buffered saline; sEVs: Small extracellular vesicles; sEV-AT: sEVs derived from the adipose tissues; sEV-LT: sEVs derived from the lipoma tissues; TEM: Transmission electron microscopy

\section{Acknowledgements}

We thank and appreciate Zhigang Yao, Long Li, Gui Liu, Lin Cui, and Xinjia Cai's (Department of Oral Pathology, Xiangya Stomatological Hospital, Central South University, China) assistance in the interpretation and preparation of the histological section.

\section{Authors' contributions}

Pengyu Hong, Kun Li, and Zhangui Tang designed the experiment; Pengyu Hong performed the experiments, analyzed the data, and wrote the manuscript; Xiaoyang Xu and Xin Hu discussed the research designs and results; Hao Yang, Yue Wu, and Juan Chen revised the manuscript. All authors read and approved the final manuscript.

\section{Funding}

This work has been supported by the National Natural Science Foundation of China (81800952), National Natural Science Foundation of China (81671003), the Natural Science Foundation of Hunan Province, China (2018JJ3712), and the Fundamental Research Funds for the Central Universities of Central South University (2018zzts838).

\section{Availability of data and materials}

The data that support the findings of this study are available from the corresponding author upon reasonable request.

\section{Declarations}

\section{Ethics approval and consent to participate}

The study was approved by the Ethical Committees of the Department of Laboratory Animals, Central South University, China (approvals no.

2020sydw0045), and the Ethical Committees of the Xiangya Stomatological Hospital \& School of Stomatology, Central South University, China (approvals no. 20190058).

\section{Consent for publication}

Not applicable.

\section{Competing interests}

The authors declare that they have no competing interests.

\section{Author details}

'Department of Oral \& Maxillofacial Surgery, Xiangya Stomatological Hospital \& School of Stomatology, Central South University, Changsha 410008, Hunan, China. ${ }^{2}$ Department of Oral and Maxillofacial Surgery, Xiangya Hospital, Central South University, Changsha, China. 
Received: 7 January 2021 Accepted: 15 March 2021

Published online: 31 March 2021

\section{References}

1. Li F, Guo W, Li K, Yu M, Tang W, Wang H, Tian W. Improved fat graft survival by different volume fractions of platelet-rich plasma and adipose-derived stem cells. Aesthet Surg J. 2015;35(3):319-33. https://doi.org/10.1093/asj/ sju046.

2. Mizuno $\mathrm{H}$. Adipose-derived stem cells for regenerative medicine in the field of plastic and reconstructive surgery. J Oral Biosci. 2013;55(3):132-6. https:// doi.org/10.1016/j.job.2013.04.005.

3. Neuber F. Fettransplantation. Chir Kongr Verhandl Dsch Gesellsch Chir. 1893; 22:66.

4. Argentati C, Morena F, Bazzucchi M, Armentano I, Emiliani C, Martino S. Adipose stem cell translational applications: from bench-to-bedside [J]. Int J Mol Sci. 2018;19(11):3475. https://doi.org/10.3390/ijms19113475.

5. Zijing $L$, Yi, et al. Adipose tissue extract promotes adipose tissue regeneration in an adipose tissue engineering chamber model. Cell Tissue Res. 2016;364(2):289-98. https://doi.org/10.1007/s00441-015-2322-5.

6. Ntege $\mathrm{EH}$, Sunami $\mathrm{H}$, Shimizu Y. Advances in regenerative therapy: a review of the literature and future directions. Regen Ther. 2020;14:136-53. https:// doi.org/10.1016/j.reth.2020.01.004

7. Li K, Li F, Li J, Wang H, Zheng X, Long J, Guo W, Tian W. Increased survival of human free fat grafts with varying densities of human adipose-derived stem cells and platelet-rich plasma. J Tissue Eng Regen Med. 2017;11(1): 209-19. https://doi.org/10.1002/term.1903.

8. Gomillion CT, Burg KJL. Stem cells and adipose tissue engineering Biomaterials. 2006;27(36):6052-63. https://doi.org/10.1016/j.biomaterials.2006. 07.033.

9. Kim N, Cho S-G. New strategies for overcoming limitations of mesenchymal stem cell-based immune modulation. Int J Stem Cells. 2015;8(1):54-68. https://doi.org/10.15283/ijsc.2015.8.1.54

10. Kusuma GD, Carthew J, Lim R, Frith JE. Effect of the microenvironment on mesenchymal stem cell paracrine signaling: opportunities to engineer the therapeutic effect. Stem Cells Dev. 2017;26(9):617-31. https://doi.org/10.1 089/scd.2016.0349

11. Thery C, Witwer KW, Aikawa E, et al. Minimal information for studies of extracellular vesicles 2018 (misev2018): a position statement of the international society for extracellular vesicles and update of the misev2014 guidelines. J Extracell Vesicles. 2018;7(1):1535750. https://doi.org/10.1080/2 0013078.2018 .1535750

12. Guo W, Li Y, Pang W, Shen H. Exosomes: a potential therapeutic tool targeting communications between tumor cells and macrophages. Mol Ther. 2020;28(9):1953-64. https://doi.org/10.1016/j.ymthe.2020.06.003.

13. van der Pol E, Boing AN, Harrison P, et al. Classification, functions, and clinical relevance of extracellular vesicles. Pharmacol Rev. 2012;64(3):676705. https://doi.org/10.1124/pr.112.005983

14. Raposo G, Stoorvogel W. Extracellular vesicles: exosomes, microvesicles, and friends. J Cell Biol. 2013;200(4):373-83. https://doi.org/10.1083/jcb.20121113 8.

15. Ge Q, Xie XX, Xiao X, et al. Exosome-like vesicles as new mediators and therapeutic targets for treating insulin resistance and $\beta$-cell mass failure in type 2 diabetes mellitus. J Diabetes Res. 2019;2019:3256060.

16. Hong P, Yang H, Wu Y, Li K, Tang Z. The functions and clinical application potential of exosomes derived from adipose mesenchymal stem cells: a comprehensive review. Stem Cell Res Ther. 2019;10(1):242. https://doi.org/1 0.1186/s13287-019-1358-y.

17. Zhang Y, Yu M, Dai M, Chen C, Tang Q, Jing W, Wang H, Tian W. Mir-450a$5 p$ within rat adipose tissue exosome-like vesicles promotes adipogenic differentiation by targeting wisp2. J Cell Sci. 2017;130(6):1158-68. https:// doi.org/10.1242/jcs.197764

18. Dai M, Yu M, Zhang Y, Tian W. Exosome-like vesicles derived from adipose tissue provide biochemical cues for adipose tissue regeneration. Tissue Eng A. 2017;23(21-22):1221-30. https://doi.org/10.1089/ten.tea.2017.0045.

19. Amundson JR, Straus D, Azab B, Liu S, Garcia Buitrago MT, Yakoub D. Giant symptomatic gastric lipoma: a case report and literature review. Int J Surg Case Rep. 2018;51:313-7. https://doi.org/10.1016/j.ijscr.2018.08.061.

20. Zavan B, De Francesco F, D'Andrea F, et al. Persistence of cd34 stem marker in human lipoma: searching for cancer stem cells. Int J Biol Sci. 2015;11(10): 1127-39. https://doi.org/10.7150/ijbs.11946.
21. Suga $H$, Eto H, Inoue K, Aoi N, Kato H, Araki J, Higashino T, Yoshimura K. Cellular and molecular features of lipoma tissue: comparison with normal adipose tissue. Br J Dermatol. 2009;161(4):819-25. https://doi.org/10.1111/ j.1365-2133.2009.09272.x

22. Halbleib M, Skurk T, Luca CD, et al. Tissue engineering of white adipose tissue using hyaluronic acid-based scaffolds. I: in vitro differentiation of human adipocyte precursor cells on scaffolds. Biomaterials. 2003;24(18): 3125-32. https://doi.org/10.1016/S0142-9612(03)00156-X.

23. Wu I, Nahas Z, Kimmerling KA, Rosson GD, Elisseeff JH. An injectable adipose matrix for soft-tissue reconstruction. Plast Reconstr Surg. 2012; 129(6):1247-57. https://doi.org/10.1097/PRS.0b013e31824ec3dc.

24. Sarkanen JR, Kaila V, Mannerstrom B, et al. Human adipose tissue extract induces angiogenesis and adipogenesis in vitro. Tissue Eng Part A. 2012 18(1-2):17-25. https://doi.org/10.1089/ten.tea.2010.0712.

25. Zhang Y, Yu M, Tian W. Physiological and pathological impact of exosomes of adipose tissue. Cell Prolif. 2016;49(1):3-13. https://doi.org/10.1111/ cpr.12233.

26. Sano S, Izumi Y, Yamaguchi T, Yamazaki T, Tanaka M, Shiota M, Osada-Oka M, Nakamura Y, Wei M, Wanibuchi H, Iwao H, Yoshiyama M. Lipid synthesis is promoted by hypoxic adipocyte-derived exosomes in 3t3-11 cells. Biochem Biophys Res Commun. 2014;445(2):327-33. https://doi.org/10.1016/ j.bbrc.2014.01.183.

27. Stojanovic S, Najman S, Korac A. Stem cells derived from lipoma and adipose tissue-similar mesenchymal phenotype but different differentiation capacity governed by distinct molecular signature. Cells. 2018;7(12):260. https://doi.org/10.3390/cells7120260.

28. Stojanović S, Najman SA-O. The effect of conditioned media of stem cells derived from lipoma and adipose tissue on macrophages' response and wound healing in indirect co-culture system in vitro. Int J Mol Sci. 2019; 20(7):1671.

29. Arnhold S, Elashry MA-O, Klymiuk MC, et al. Investigation of stemness and multipotency of equine adipose-derived mesenchymal stem cells (ascs) from different fat sources in comparison with lipoma. Stem Cell Res Ther. 2019;10(1):309. https://doi.org/10.1186/s13287-019-1429-0.

30. Xie $Y$, Chen $Y$, Zhang $L, G e ~ W$, Tang $P$. The roles of bone-derived exosomes and exosomal micrornas in regulating bone remodelling. J Cell Mol Med. 2017:21(5):1033-41. https://doi.org/10.1111/jcmm.13039.

31. Ching RC, Kingham PJ. The role of exosomes in peripheral nerve regeneration. Neural Regen Res. 2015;10(5):743-7. https://doi.org/10.4103/1 673-5374.156968.

32. Ji SC, Yoon HI, Lee KS, et al. Exosomes from differentiating human skeletal muscle cells trigger myogenesis of stem cells and provide biochemical cues for skeletal muscle regeneration. J Control Release. 2016;222:107-15.

33. Neels JG, Thinnes T Fau - Loskutoff DJ, Loskutoff DJ. . FASEB J, 2004, 18(9): 983-985, DOl: https://doi.org/10.1096/fj.03-1101fje.

34. Cao Y. Adipose tissue angiogenesis as a therapeutic target for obesity and metabolic diseases. Nat Rev Drug Discov. 2010;9(2):107-15. https://doi.org/1 $0.1038 / \mathrm{nrd} 3055$

\section{Publisher's Note}

Springer Nature remains neutral with regard to jurisdictional claims in published maps and institutional affiliations.

Ready to submit your research? Choose BMC and benefit from:

- fast, convenient online submission

- thorough peer review by experienced researchers in your field

- rapid publication on acceptance

- support for research data, including large and complex data types

- gold Open Access which fosters wider collaboration and increased citations

- maximum visibility for your research: over $100 \mathrm{M}$ website views per year

At BMC, research is always in progress.

Learn more biomedcentral.com/submissions 